\title{
A novel mutation in a case of pseudohypoparathyroidism type Ia
}

\author{
Birgül Kırel ${ }^{1}$, Meliha Demiral ${ }^{1}$, Özkan Bozdağ ${ }^{2}$, Kadri Karaer ${ }^{3}$ \\ ${ }^{1}$ Division of Pediatric Endocrinology, ${ }^{2}$ Department of Pediatrics, Osmangazi University Faculty of Medicine, Eskişehir, \\ ${ }^{3}$ Department of Clinical Genetics, Dr. Ersin Arslan Hospital, Gaziantep, Turkey \\ E-Mail: birkirel9@gmail.com
}

Received: 17 February 2015, Revised: 3 June 2015, Accepted: 14 July 2015

\begin{abstract}
SUMMARY: Kurel B, Demiral M, Bozdağ Ö, Karaer K. A novel mutation in a case of pseudohypoparathyroidism type Ia. Turk J Pediatr 2016; 58: 101-105.

Pseudohypoparathyroidism (PHP) type Ia is characterized by multiple hormone resistance; primarily parathyroid hormone (PTH) resistance and Albright's hereditary osteodystrophy (AHO) which involves skeletal and developmental defects. The AHO phenotype alone without hormone resistance is defined as pseudoPHP. A boy was first diagnosed as having both rickets and primary hypothyroidism at 2.5 months of age. His calcium level remained within normal levels after vitamin D treatment, but, elevated PTH and ALP levels and normal-high phosphate levels persisted during his follow-up by age of 2.5 years. He was admitted with hypocalcemic convulsions as well as hyperphosphatemia and elevated PTH levels suggested PTH resistance at 2.5 years of age. He and his mother were obese and had round faces, frontal bossing, small noses, flat nasal bridges, brachydactyly. His mother showed no hormonal resistance. These findings indicated that our patient had PHP type Ia and his mother had pseudoPHP. The same novel heterozygous mutation in the GNAS gene (IVS4+5G>C) was identified in both of patients.
\end{abstract}

Key words: pseudohypoparathyroidism, Albright's hereditary osteodystrophy, children, novel mutation, GNAS gene.

Hormone resistance, a condition presenting a low and normal end-organ hormone levels and an elevated stimulatory hormone levels, is the result of defects in the structure and functions of the target receptor and intracellular signaling system. Activation of the alphasubunit of the guanine nucleotide-binding protein (Gsa) by a hormone receptor leads to stimulation of adenyl cyclase which plays an important role in the cellular response of many hormones through the generation of cyclic AMP (cAMP) ${ }^{1,2}$. Gsa activity is required for many physiological cellular events ${ }^{1}$. The GNAS gene complex (20q13) encodes the transcripts of this protein and several other products involved in the actions of specific hormones ${ }^{1,2}$. This gene is expressed biallelically in most tissues; on the other hand, expression of Gsa and other products of the GNAS gene locus in some tissues is determined by genomic imprinting according to the their parental origin ${ }^{2}$. Heterogeneity in clinical and laboratory findings of the same mutations in the GNAS gene locus mainly reflects whether the mutation is inherited maternally or paternally ${ }^{1,2}$. Maternal heterozygous inactivating mutations or epimutations of the GNAS gene locus cause a rare inherited genetic disorder with variable clinical presentations where some present with multiple hormone resistance in target tissues such as the renal proximal tubules, pituitary gland, thyroid and gonads, in which the latter gene locus is maternally imprinted.

An important variant of the disorder is associated with multiple hormone resistance; especially parathyroid hormone (PTH) resistance known as pseudohypoparathyroidism (PHP). Inactivating mutations of GSa encoded by the exons 1-13 of the GNAS gene lead to PHP type Ia and Albright's hereditary osteodystrophy 
(AHO) phenotype which involves skeletal and developmental defects when it is on the maternal allele. When the same mutation is inherited paternally, it causes only the AHO phenotype defined as pseudoPHP and does not cause any hormone resistance ${ }^{1,2}$.

In the present report: a 5-year-old boy with PHP type Ia and his mother had the AHO phenotype alone are discussed. A novel heterozygous mutation (IVS4+5G $>$ C) in the GNAS gene was identified in both of patients.

\section{Case Report}

Our patient, a male baby was born at term gestation with a birth weight of $3,100 \mathrm{~g}$ to a 27-year-old primagravida mother by caesarean section due to dystocia. The parents reported no consanguinity. Prophylactic vitamin D (400 units/day) was initiated on the $14^{\text {th }}$ day after birth. The patient was breastfed alone until 6 months of age and was hospitalized for pneumonia in the pediatric intensive care unit (ICU) at 2.5 months of age. Upon physical examination, body weight (BW) was $6,200 \mathrm{~g}$, height was $56 \mathrm{~cm}$, relative weight was $134 \%$ and a coarse face and umbilical hernia were observed. Laboratory investigations were as follows: blood calcium $(\mathrm{Ca}): 7.0 \mathrm{mg} / \mathrm{dl}$, phosphate $(\mathrm{P}): 3.84 \mathrm{mg} /$ $\mathrm{dl}$ and alkaline phosphatase (ALP): $622 \mathrm{U} / \mathrm{L}$ (0-462) and PTH: $236 \mathrm{pg} / \mathrm{ml}$ (11-67). He was diagnosed with rickets due to vitamin $\mathrm{D}$ deficiency. Radiographic findings of rickets were not shown on the X-ray of hand-wrist. After intravenous calcium (Ca) treatment, he was administrated oral calcium and oral vitamin D3 (1000 IU/ day). Ca treatment was discontinued after $\mathrm{Ca}$ levels reached normal levels, and a prophylactic dose of vitamin D was administrated again 8 weeks later. At that time, L-thyroxin supplementation was initiated because of hypothyroidism (TSH: $17.5 \mu \mathrm{IU} /$ $\mathrm{ml}$ and fT4: $0.59 \mathrm{ng} / \mathrm{dl})$. He was operated on for right inguinal hernia at four months of age. He was followed-up at 3-month intervals for primary hypothyroidism by our outpatient clinic. During these follow-ups, although his $\mathrm{Ca}$ levels were within normal levels, elevated PTH and ALP levels together with normal-high phosphate levels were observed on several visits (Fig. 1). No further analysis or treatments were performed for these abnormalities.
At 2.5 years of age, when he was admitted with fever and convulsions to our emergency department, hypocalcemia was observed. Upon physical examination, we observed BW: $16 \mathrm{~kg}$ (90-97 p), height: $87 \mathrm{~cm}$ (10-25 p), relative weight: $120 \%$ and body mass index (BMI): 21.3 $\mathrm{kg} / \mathrm{m}^{2}$ (>95 p). He had round face, depressed nasal bridge, anteverted nostrils, frontal bossing and brachydactyly. Laboratory tests revealed Ca: $4.9 \mathrm{mg} / \mathrm{dl}$, P: $9.8 \mathrm{mg} / \mathrm{dl}$, ALP: $546 \mathrm{U} / \mathrm{L}$ (0-281), PTH: $363 \mathrm{pg} / \mathrm{ml}$ (11-67 pg/ml), creatinine: $0.38 \mathrm{mg} / \mathrm{dl}$, and spot urine $\mathrm{Ca} / \mathrm{creatinine:} 0.06$; renal ultrasonography was normal. 25-Hydroxyvitamin $\mathrm{D}$ level analysis was not available at that time in our hospital. X-rays of the hand-wrist and cranium revealed no pathological findings. No ectopic ossifications were observed either clinically or on X-ray. Upon admission, he was evaluated as having PTH resistance as well as mild TSH resistance and the AHO phenotype;
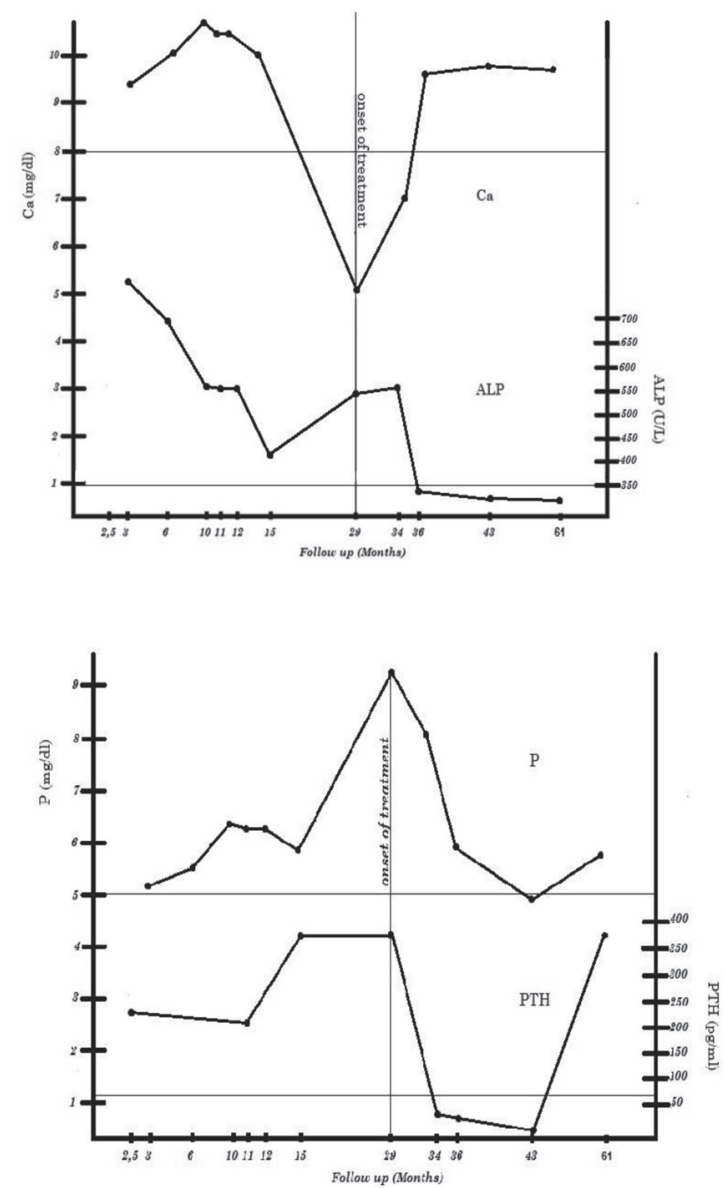

Fig.1. The follow-up of calcium metabolism parameters of the patient. 
thus, the diagnosis was PHP type Ia. After intravenous calcium treatment, he was put on regular calcitriol and $\mathrm{Ca}$ supplementation. During his follow-up, normocalcemia remained and P, ALP and PTH levels decreased to within normal limits. The patient is now 5 years old. Within the last few months, bilateral minimal renal pelvic dilatation was detected by ultrasonography. Vesicoureteral reflux (VUR) grade 3 on the right side and grade 4 on the left side was detected based on voiding cystourethrography, and he was operated for VUR. His renal function tests were within normal limits. Upon his last admission, his measurements were as follows: BW: $26 \mathrm{~kg}$ (>97 p), height: $108 \mathrm{~cm}$ (25 p), BMI: 25.7 $\mathrm{kg} / \mathrm{m}^{2}$ (>95 p), Ca: $9.8 \mathrm{mg} / \mathrm{dl}, \mathrm{P}: 6 \mathrm{mg} / \mathrm{dl}$, ALP: $158 \mathrm{U} / 1$ (0-269), PTH: 363 pg/ml (1167), BUN: $9.3 \mathrm{mg} / \mathrm{dl}$, and creatinine: $0.3 \mathrm{mg} /$ dl. His mental status was evaluated as normal.

The patient's mother was obese (BW: 64 $\mathrm{kg}$, BMI: $30.4 \mathrm{~kg} / \mathrm{m}^{2}$ ) and had a round face, depressed nasal bridge, anteverted nostrils, brachydactyly and short stature $(148 \mathrm{~cm})$. The mother's parents had no any dysmorphic features. No abnormalities in thyroid function tests and $\mathrm{Ca}$ metabolism parameters were observed in either the mother or her parents. Thus, the mother had an AHO phenotype and was diagnosed as having pseudoPHP.

We performed targeted next generation sequencing (NGS) of the related GNAS gene. Informed consent from the whole family was obtained prior to collection of $5 \mathrm{ml}$ of peripheral blood for the following experiments. Sequencing analysis revealed a novel heterozygous mutation (IVS4+5G $>$ C) in the GNAS gene $(20 \mathrm{q} 13,3)$, which was identified in both the patient and his mother. The nucleotide $G$ is highly conserved at position 5 of the consensus donor splice sites of introns. Transversion of $\mathrm{G}$ to $\mathrm{C}$ at this position has been associated with abnormal splicing and severe gene expression defects in a number of genes. The putative splicing alterations caused by splice site mutations were predicted using the websites including Human splicing finder and Splice Port ${ }^{3,4}$. A novel heterozygous mutation (IVS $4+5 \mathrm{G}>\mathrm{C}$ ) in the GNAS gene $(20 \mathrm{q} 13,3)$ was identified in both the patient and his mother. No mutations were observed in the father.

\section{Discussion}

Pseudohypoparathyroidism is caused mostly by maternal heterozygous inactivating mutations in the GNAS gene, leading to a defect in the signaling pathway of $G$ protein-coupled receptors that results in resistance to certain hormones such as PTH, TSH, FSH, LH and growth hormone releasing hormone. The most common resistance is observed for $\mathrm{PTH}^{1,5}$. Our patient had both PTH and TSH resistance. GNAS gene expression at the target tissues of these hormones is determined by maternal imprinting 1,2 .

The type and parental origin of the GNAS gene defects are responsible from different clinical variants of the disease such as the AHO phenotype, progressive osseous heteroplasia $(\mathrm{POH})$, and PHP. Maternal inheritance of a mutation in the GNAS gene affecting exons 1-13 likely leads to the PHP Ia phenotype. However, the paternal inheritance of the same mutation typically results in the AHO phenotype, defined as pseudoPHP1,2,5.

The AHO phenotype is a group of physical characteristics with variable prevalence including stocky build; short stature and obesity, round face, mental retardation, brachydactyly and ectopic ossifications 1,5. Gsa haploinsufficiency is thought to play a primary role in the development of the AHO phenotype ${ }^{2}$. Our patient and his mother had most of the physical features of the AHO phenotype. The mother showed no hormone resistance and she was diagnosed with pseudoPHP.

A novel heterozygous mutation in the GNAS gene was identified in both our patient and his mother. This finding supports the conclusions that PHP Ia type is maternally transmitted. On the other hand, the same mutation in our patient's mother with pseudoPHP could have been inherited paternally or a de novo mutation. We did not perform genetic analysis on the mother's parents.

Pseudohypoparathyroidism is classified according to the presence of the AHO phenotype, multiple hormone resistance and response to exogenous PTH administration ${ }^{1,5}$. PHP type I patients show both blunted renal CAMP generation and phosphaturic responses to exogenous PTH administration. In PHP type II patients, this phosphaturic response is 
impaired, however, both basal and stimulated renal cAMP responses are preserved during this test ${ }^{1,2}$. The AHO phenotype is not observed in patients with PHP type II. PHP type I is divided into three subtypes. PHP types Ia and Ic patients have both the AHO phenotype and multiple hormone resistance, as seen in our patient. PHP Ib patients show only isolated hormone resistance without the AHO phenotype. Gsa bioactivity is normal in PHP $\mathrm{Ib}$ and Ic, but is decreased in PHP Ia ${ }^{1,7}$. PHP types Ia and Ic are not clinically different conditions. PHP Ic is caused by receptor coupling defects leading to the same signaling defect as in PHP Ia ${ }^{1}$. It has been reported that PHP Ib is more commonly caused by epigenetic imprinting abnormalities affecting control elements regulating methylation of the GNAS gene and its expression rather than by GNAS gene mutations ${ }^{1,7}$.

It has been reported that obesity and prevalence of extreme obesity are twofold and 3.5 fold higher, respectively, in adults with PHP Ia. Obesity is more common in PHP Ia patients than in pseudoPHP patients. Our patient was obese from early infancy, and his mother was also obese.

The prevalence of mental retardation is $47-75 \%$ in the PHP I patients; however, the cause of this remains unknown ${ }^{9}$. No mental retardation was observed in our patient.

Progressive osseous heteroplasia is another rare, clinical variant of PHP characterized by progressive soft tissue ossifications in dermis, muscle and deep connective tissue. It is not associated with the AHO phenotype or hormone resistance. The paternal inheritance heterozygous inactivating mutations of the GNAS gene results in the $\mathrm{POH}$ phenotype ${ }^{1,6}$. Our patient and his mother did not have $\mathrm{POH}$.

In our patient at 2.5 months of age, Ca levels are low and $\mathrm{P}$ levels are normal as seen in the early stages of rickets due to vitamin $\mathrm{D}$ deficiency. Thus, he had been diagnosed with rickets. Maternal vitamin D deficiency during the intrauterine and lactation periods is the major contributing factor for rickets development during early infancy in our country $^{10}$. We did not determine 25-hydroxyvitamin D levels in either the patient or his mother. In patients with rickets, $\mathrm{P}$ levels then decrease due to renal excretion if vitamin $\mathrm{D}$ treatment is not administrated. On the other hand, hyperphosphatemia is not a common finding in vitamin $\mathrm{D}$ deficiency, although it may be found in some patients with rickets due to the presence of partial PTH resistance mediated by PTH itself ${ }^{11}$. Thus, early vitamin D deficiency could be confused with PHP in patients with normal renal functions. Differential diagnosis is relatively simple if 25-hydroxy-vitamin D levels are analyzed. Normalization of all calcium metabolism parameters after vitamin D3 treatment may also be helpful for differentation ${ }^{12}$. In our patient, hyperphosphatemia and elevated ALP and PTH levels were observed several times during follow-up visits until 2.5 years of age. No further analysis or treatment was performed for these abnormalities. In our patient, abnormalities in $\mathrm{Ca}$ metabolism parameters until 2.5 years of age were not compatible with the clinical course of nutritional rickets. Thus, our patient was diagnosed with PTH resistance as well as PHP type Ia because of the AHO phenotype. Primary hypothyroidism in our patient was suggestive of the presence of TSH resistance and supported our diagnosis.

It has been reported that all PHP Ia patients present with hypocalcemia and the onset of PTH resistance typically occurs after early childhood $^{2}$. Gelfand et al. ${ }^{5}$ analyzed medical reports of 12 patients with PHP and reported that hypothyroidism and subcutaneous ossifications were the most common presenting symptoms in the first 2 years of life. Elevated PTH was observed in $42 \%$ of patients during infancy period. However, PTH resistance developed in all patients by the second decade of life. These reported findings are similar to our patient's clinical course.

In patients with PHP, PTH resistance was observed only in proximal tubules. There was no resistance to the actions of PTH in bone or thick ascending tubule. Thus, as in our patient, elevated PTH levels could maintain normocalcemia for extended times ${ }^{1}$. On the other hand, beside normocalcemia, persistent elevated ALP and PTH levels are indicative of a hyper-metabolic bone disease, as in our patient. Treatment of patients is advised to prevent bone abnormalities caused by elevated $\mathrm{PTH}^{1}$.

In conclusion, we report a novel heterozygous mutation in the GNAS gene leading to PHP 
type Ia in a child and pseudoPHP in his mother. In the future, clinicians should consider that PHP could be confused with rickets. We believe that this case report will contribute to the diagnosis of this clinically and genetically heterogeneous disease.

\section{REFERENCES}

1. Bastepe M, Jüppner H. GNAS locus and pseudohypoparathyroidism. Horm Res 2005; 63: 65-74.

2. Turan S, Bastepe MT. The GNAS complex locus and human diseases associated with loss-of-function mutations or epimutations within this imprinted gene. Horm Res Paediatr 2013; 80: 229-241.

3. Desmet FO, Hamroun D, Lalande M, Et al. Human splicing finder: an online bioinformatics tool to predict splicing signals. Nucleic Acids Res 2009; 37: e67.

4. Dogan RI, Getoor L, Wilbur WJ, Mount SM. SplicePortan interactive splice-site analysis tool. Nucleic Acids Res 2007; 35: 285-291.

5. Gelfand IM, Eugster EA, DiMeglio LA. Presentation and clinical progression of pseudohypoparathyroidism with multi-hormone resistance and Albright hereditary osteodystrophy: a case series. J Pediatr 2006; 149: 877-880.
6. Gelfand IM, Hub RS, Shore EM. Kaplan FS, Dimeglio LA. Progressive osseous heteroplasialike heterotopic ossification in a male infant with pseudohypoparathyroidism type Ia: a case report. Bone 2007; 40: 1425-1428.

7. Mariot V, Maupetit-Méhouas S, Sinding C, Kottler ML, Linglart A. A maternal epimutation of GNAS leads to Albright osteodystrophy and parathyroid hormone resistance. J Clin Endocrinol Metab 2008; 93: 661-665.

8. Long DN, McGuire S, Levine MA, Weinstein LS, Germain-Lee EL. Body mass index differences in pseudohypoparathyroidism type la versus pseudopseudohypoparathyroidism may implicate paternal imprinting of $\mathrm{G}$ alpha(s) in the development of human obesity. J Clin Endocrinol Metab 2007; 92: 1073-1079.

9. Farfel ZVI, Friedman E. Mental deficiency in pseudohypoparathyroidism Type I is associated with Ns-protein deficiency. Ann Intern Med 1986; 105: 197-199.

10. Hatun S, Ozkan B, Orbak Z, Et al. Vitamin D deficiency in early infancy. J Nutr 2005; 135: 279-282.

11. Allgrove J. A practical approach to rickets. In: Allgrove J; Shaw NJ (eds). Calcium and bone disorders in children and adolescents; Endocr Dev Vol.16. Basel, 2009; 115-132.

12. Akın L, Kurtoğlu S, Kendirci M. Vitamin D deficiency rickets mimicking pseudohypoparathyroidism. J Clin Res Pediatr Endocrinol 2010; 2: 173-175. 